\title{
Flame retardant and mechanical properties of phenolic foams toughened with phosphorus- and boron- containing polyethylene glycol esters
}

\author{
Yuxiang Zhu ${ }^{a}$ and Zhengzhou Wang * \\ School of Materials Science and Engineering, Tongji University, Shanghai, China, 200092 \\ a1333637@tongji.edu.cn, bzwang@tongji.edu.cn
}

\begin{abstract}
Keywords: Toughening, Phenolic foam, Boron and phosphorus containing polyesters
Abstract. Phosphorus and boron containing polyethylene glycol esters (PB-PEG) toughening agent was synthesized by the esterification of phosphorus pentaoxide $\left(\mathrm{P}_{2} \mathrm{O}_{5}\right)$, boric acid and polyethylene glycol (PEG). Phenolic foams toughened with PB-PEG were prepared. It is found that the flexural and compression strength of the foams increases with the addition of PB-PEG. Optical images of the foams show that the addition of the toughening agents can make the cells uniform and regular. LOI results indicate that the incorporation of PB-PEG has positive effect on the fire resistance of the foams. Moreover, pulverization ratio of the toughened foams was reduced.
\end{abstract}

\section{Introduction}

In recent years, phenolic (PF) foams have attracted much attention due to their excellent flame retardant properties compare with polyurethane (PU) and polystyrene (PS) foams in the field of building insulation materials. ${ }^{[1]}$ However, PF foams have some serious shortcomings such as brittleness and pulverization which greatly limit its applications in some cases. ${ }^{[2]}$ Thus, many researchers have focused their attention on the toughening and strengthening of phenolic foams. ${ }^{[3]}$ There are generally two kinds of modifying methods for phenolic foams: chemical modification and physical modification. For example, Jing et al. applied reactive monomers similar to phenol containing flexible segments (e.g. cardanol) to partly replace phenol in order to produce a phenolic resin with flexible chains in its rigid backbones. ${ }^{[4]}$ The polymeric toughening agents, such as polyethylene glycol (PEG) and polyvinyl alcohol (PVA) are usually incorporated during the preparation of the phenolic foams to enhance their toughness. ${ }^{[5,6]}$ However, the commonly used polymeric toughening agents are very flammable resulting in a decrease in flame retardancy of PF foams.

In this study, phosphorus and boron containing polyethylene glycol ester (PB-PEG) toughening agents were synthesized through the reaction of $\mathrm{P}_{2} \mathrm{O}_{5}$, boric acid and PEG. The effect of PB-PEG on mechanical and flame retardant properties of PF foams was evaluated.

\section{Experimental}

\section{Materials}

\section{Synthesis of flame retardant toughening agents}

PEG400 (80g, 0.200mol) was put into a 500-ml three-neck flask with a mechanical stirrer, and the flask was heated to $50^{\circ} \mathrm{C} . \mathrm{P}_{2} \mathrm{O}_{5}(4.73 \mathrm{~g}, 0.033 \mathrm{~mol})$ was added into the aforementioned flask slowly with an approximate $1 \mathrm{~h}$ under strong agitation. After $\mathrm{P}_{2} \mathrm{O}_{5}$ was dispersed evenly, the mixture was heated to $120^{\circ} \mathrm{C}$ and then stirred for another $3 \mathrm{~h}$. After that, boric acid $(4.13 \mathrm{~g}, 0.067 \mathrm{~mol})$ was added into the mixture and heated for $3 \mathrm{~h}$. Finally, the mixture was cooled to room temperature, and a light yellow viscous liquid was obtained.

\section{Preparation of phenolic foams}

A certain proportion of resol-type phenolic resin, Tween-80 (surfactant) and PB-PEG (toughening agent) were added into a mixer at room temperature. The mixture was stirred strongly. The curing agent (phosphoric acid: p-toluenesulfonic acid: water $=1: 2: 2$, mass ratio) and foaming agent (n-pentane) were added into the mixer at room temperature and then stirred strongly and quickly. The 
obtained viscous mixture was poured into the mould and cured at $80^{\circ} \mathrm{C}$ for $1 \mathrm{~h}$. To keep the density of the foam $\left(16 \pm 0.5 \mathrm{~kg} / \mathrm{m}^{3}\right)$ the same, the same amount of the mixture was put into the mould. The obtained foam was cut carefully for the tests. The formulations of phenolic foams were summarized in Table 1.

Table 1 Formulations of phenolic foams

\begin{tabular}{cccc}
\hline Sample code & PF resin (phr) & PB-PEG (phr) & PEG (phr) \\
\hline PF & 100 & 0 & 0 \\
PFPBPEG-2 & 100 & 2 & 0 \\
PFPBPEG-4 & 100 & 4 & 0 \\
PFPBPEG-6 & 100 & 6 & 0 \\
PFPBPEG-8 & 100 & 8 & 0 \\
PFPBPEG-10 & 100 & 10 & 0 \\
PFPEG-2 & 100 & 0 & 2 \\
PFPEG-4 & 100 & 0 & 4 \\
PFPEG-6 & 100 & 0 & 6 \\
PFPEG-8 & 100 & 0 & 8 \\
PFPEG-10 & 100 & 0 & 10 \\
\hline
\end{tabular}

Blowing agent: 8 phr; Curing agent: 9 phr; Surfactant: 5 phr.

\section{Measurements}

Mechanical properties were tested by a universal testing machine. Flame retardant properties such as limiting oxygen index (LOI) was carried out by oxygen index meter, UL-94 ratings of the foams were obtained by using a horizontal and vertical burning tester. Pulverization ratio was obtained by rubbing the sample on abrasive paper under constant weight. Cell structures of the foams were observed by optical microscope.

\section{Results and discussion}

Figure 1 shows the FT-IR spectra of flame retardant toughening agent. The peak at $3419 \mathrm{~cm}^{-1}$ is the absorption of hydroxyl groups of PEG, and it decreases obviously due to the esterification reaction between acid and PEG. The peak at $1364 \mathrm{~cm}^{-1}$ is attributed to the stretching of B-O-C, which proved the existence of PEG borate. P-O-C stretching absorptions are located at $1040 \mathrm{~cm}^{-1}$ and $989 \mathrm{~cm}^{-1}$, which proved the creation of PEG phosphate. The peak at $668 \mathrm{~cm}^{-1}$ is attributed to the absorption of $\mathrm{BO}_{3}$. The existence of P-O-C and $\mathrm{B}-\mathrm{O}-\mathrm{C}$ peaks indicates that the esters have been synthesized successfully.

Figure 2 shows the flexural strength of pure and toughened foams. The strength of PB-PEG toughened foams is higher than that of the PEG toughened one. This may be due to the acidity of PB-PEG which can catalyze the curing process of phenolic resin, increase the degree of crosslinking of phenolic resin, and thus strengthen the whole structure.

In order to investigate how the toughening agents influence the structure of the foams, optical microscope was used to observe the microstructure of the foams. The results were shown in Figure 3. As we can see in the picture, pure phenolic foam cells have an average radius about $150 \mu \mathrm{m}$, while the

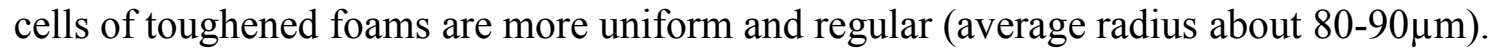




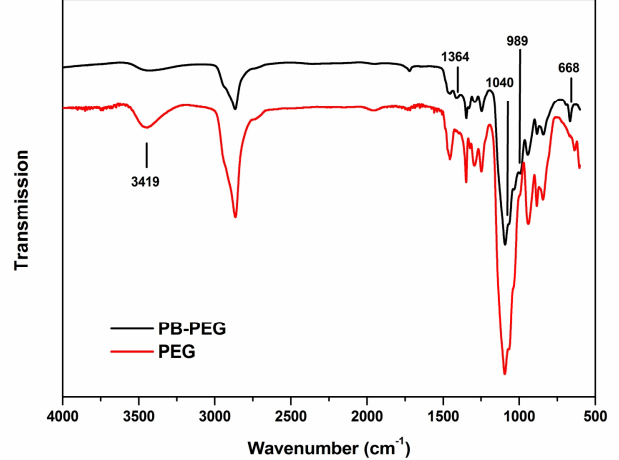

Fig. 1 FT-IR spectra of toughening agents

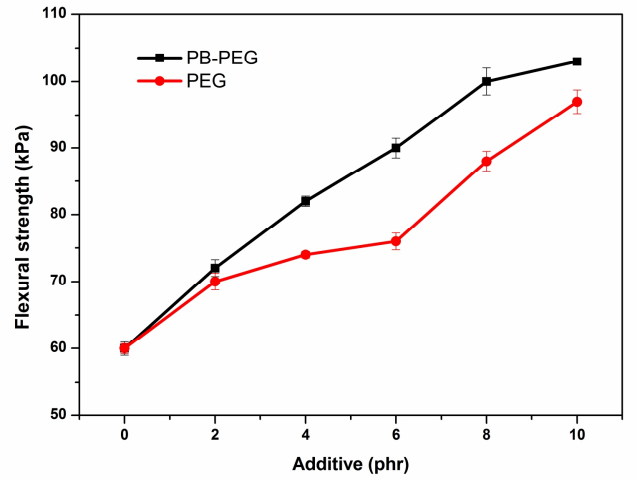

Fig. 2 Flexural strength of toughened phenolic foams
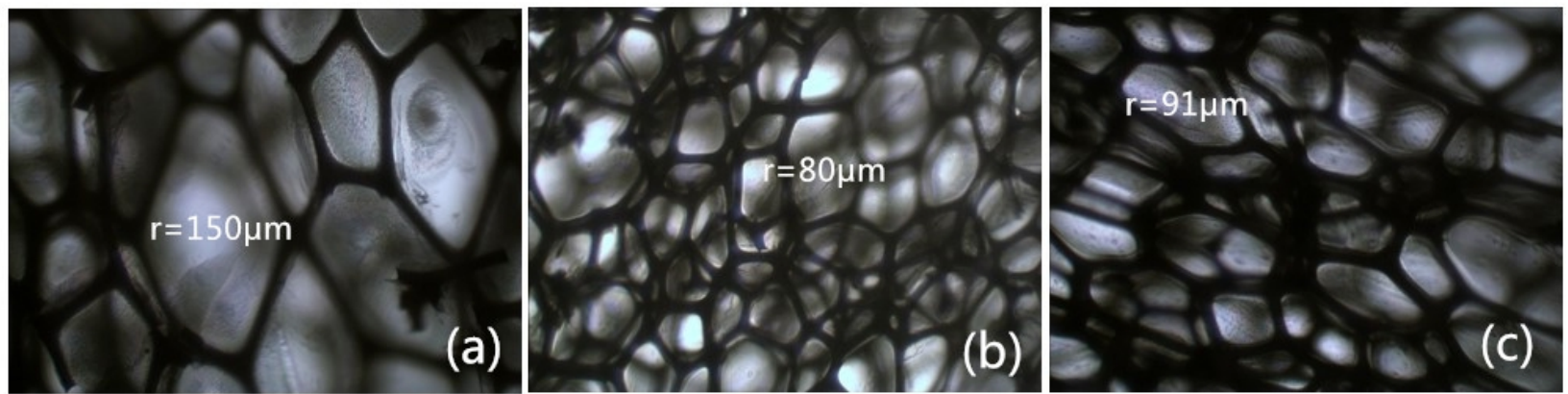

Fig. 3 Optical images, (a) pure phenolic foam; (b) PFPBPEG-6; (c) PFPEG-6

Pulverization ratio of toughened phenolic foams was presented in Fig. 4. As we can see, pulverization ratio of the samples all decreases with the increase in the content of toughening agents. PB-PEG presents a much better effect on reducing the pulverization ratio than PEG does. For example, the pulverization ratio of PB-PEG toughened foams is $4.0 \%$, while the ratio of PEG toughened foams is $5.3 \%$ at the same loading (10 phr). The values are much lower than the one of pure phenolic foam $(8.2 \%)$. This may be due to the effect of the flexible chains of the toughening agents in the foams.

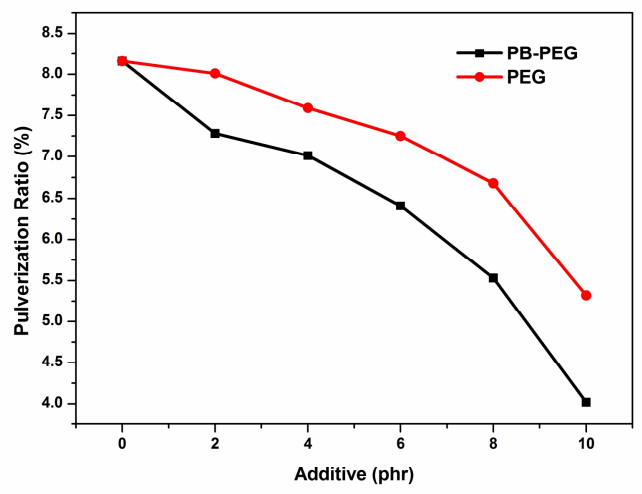

Fig. 4 Pulverization ratio of toughened phenolic foams

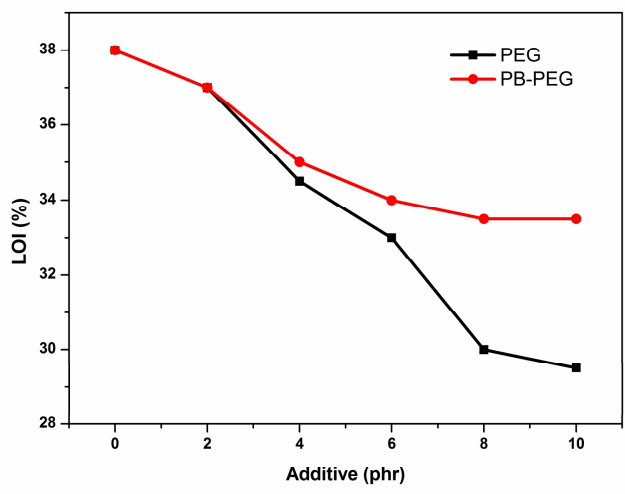

Fig. 5 LOI of toughened phenolic foams

Figure 5 presents the LOI data of pure PF foam and the toughened foams. It is obvious that the LOI of pure PF foam is 38\%. The LOI values decrease when ether PEG or PB-PEG is incorporated into the PF foam. The more the toughening agents, the lower the LOI values are. However, the LOI values of foams toughened with PB-PEG are higher than that of the foam toughened with the same addition of PEG. This demonstrates that the introduction of PB-PEG into PF foam has less influence on fire resistance of the foams. 


\section{Conclusions}

A phosphorus and boron containing polyethylene glycol ester (PB-PEG) was synthesized and used to toughen phenolic foam. Flexural strength increases with the addition of PB-PEG and PEG. Optical images show that the introduction of these agents can improve the foam structure, and make the cells uniform. LOI results indicate that the incorporation of PB-PEG has less influence on fire resistance of the foams compared with PEG. Pulverization ratio data illustrate that toughening agents have good effect in reducing pulverization of the toughened foams.

\section{Acknowledgements}

This work was financially supported by the National Natural Science Foundation of China (Nos. 21174106 and U1205114/L11).

\section{References}

[1] G. Ji, J. Wang, A Discussion on Technical Means of External Thermal Insulation and Fireproofing, Proceedings of the $7^{\text {th }}$ International Conference on Tall Buildings. (2010) 331-340.

[2] Y. J. Huang, C. H. Wang, Y. L. Huang, G. Guo and S. R. Nutt, Enhancing Specific Strength and Stiffness of Phenolic Microsphere Syntactic Foams Through Carbon Fiber Reinforcement, Polymer Composites. 31 (2010) 256-262.

[3] T. Dipl.-Ing. Gietl, H. Dr.-Ing. Lengsfeld and V. Dr.-Ing. Altstädt, The Efficiency of Various Toughening Agents in Novel Phenolic Type Thermoset Resin Systems, Journal of Materials Science. 41 (2006) 8226-8243.

[4] Shuailin Jing, Tao Li, Xiaojuan Li, Qian Xu, Jing Hu and Ruihai Li, Phenolic Foams Modified by Cardanol Through Bisphenol Modification, Journal of Applied Polymer Science. 131 (2014) 39942-39949.

[5] M. Gao, Y. L. Yang and Z. Q. Xu, Mechanical and Flame Retardant Properties of Phenolic Foam Modified with Polyethylene Glycol as Toughening Agent, Advanced Materials Research. 803 (2013) 21-25.

[6] Z. C. Fang and J. P. Suo, Synthesis and Characterization of Phenolic Resol Resin Blended with Silica and PVA, Journal of Applied Polymer Science. 119 (2011) 744-751. 